\title{
ZnO based optical modulator in the visible wavelengths
}

\author{
Ali K. Okyay*a,b Levent E. Aygun ${ }^{\mathrm{a}, \mathrm{b}}$, Feyza B. Oruc ${ }^{\mathrm{a}, \mathrm{b}}$ \\ ${ }^{a}$ Department of Electrical and Electronics Engineering, Bilkent University, Ankara 06800, Turkey; \\ ${ }^{\mathrm{b}}$ UNAM-Institute of Materials Science and Nanotechnology, Bilkent University, Ankara 06800, \\ Turkey
}

\begin{abstract}
In order to demonstrate tunable absorption characteristics of $\mathrm{ZnO}$, photodetection properties of $\mathrm{ZnO}$ based thin-filmtransistors are investigated. By controlling the occupancy of the trap states, the optical absorption coefficient of $\mathrm{ZnO}$ in the visible light spectrum is actively tuned with gate bias. An order of magnitude change of absorption coefficient is achieved. An optical modulator is proposed exploiting such tunable absorption mechanism.
\end{abstract}

Keywords: $\mathrm{ZnO}$, tunable absorber, metal oxides, thin film transistor, optical modulator, atomic layer deposition

\section{INTRODUCTION}

$\mathrm{ZnO}$ based transparent thin film transistors are extensively investigated recently due to their potential of replacing amorphous Si thin film transistors [1-5]. Also, UV (ultra-violet) detecting properties of $\mathrm{ZnO}$ photodiodes are attracting increasing attention [6-8]. Phototransistors with $\mathrm{ZnO}$ channel layer deposited by high temperature RF magnetron sputtering systems are demonstrated in the literature [9]. However, such a high temperature process is not suitable for flexible low cost substrates. Atomic layer deposition (ALD) technique can be used to deposit highly conformal $\mathrm{ZnO}$ films at low temperatures with unmatched large-area uniformity.

$\mathrm{ZnO}$ based optical modulator in ultra-violet regime is proposed by Wraback et al [10]. In this work, the optical absorption coefficient of $\mathrm{ZnO}$ channel layer in the visible spectrum actively tuned by controlling gate bias. By exploiting this absorption tuning mechanism, ALD based $\mathrm{ZnO}$ thin-film optical modulator design is proposed.

\section{METHODOLOGY}

\subsection{Fabrication and electrical characterization of $\mathrm{ZnO}$ based TFTs}

ZnO TFTs fabricated on p-type doped Si substrate with 10-18 $\mathrm{m} \Omega$-cm resistivity. Si substrate is also used as a back gate electrode. A thick $\mathrm{SiO}_{2}$ layer is deposited by PECVD to electrically isolate devices. $\mathrm{SiO}_{2}$ layer is patterned and etched to define an active channel region. As a gate oxide, 20-nm-thick $\mathrm{Al}_{2} \mathrm{O}_{3}$ is deposited using ALD at $250^{\circ} \mathrm{C}$ (precursors: trimethylaluminum and water). 10-nm-thick $\mathrm{ZnO}$ is deposited as the channel layer at $80^{\circ} \mathrm{C}$ with $\mathrm{ALD}$ technique which uses diethylzinc and water as precursors. The $\mathrm{ZnO}$ channel layer is patterned and etched using dilute $\mathrm{H}_{2} \mathrm{SO}_{4}: \mathrm{H}_{2} \mathrm{O}$ (2:98) solution. $100 \mathrm{~nm}$ thick Al source and drain contacts are deposited using a thermal evaporator and patterned with a lift-off technique. 3D schematic of ZnO TFTs and top SEM view of fabricated device are given in Figure 1.

Oxide-based Materials and Devices IV, edited by Ferechteh Hosseini Teherani, David C. Look, David J. Rogers, Proc. of SPIE Vol. 8626, 86261T · (c) 2013 SPIE · CCC code: 0277-786X/13/\$18 · doi: 10.1117/12.2005558 

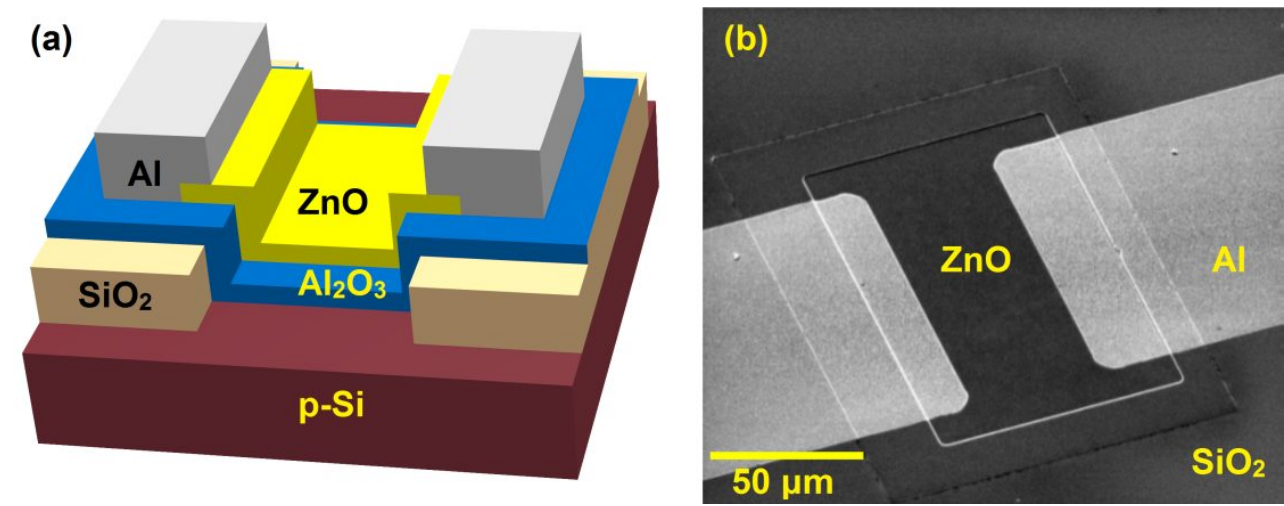

Figure 1. (a) The device schematic of ZnO TFT. (b) Tilted top view of the fabricated device.

Drain current of $\mathrm{ZnO}$ TFT measured for various gate voltage biases under constant source to drain voltage bias of $0.5 \mathrm{~V}$ are shown in Figure 2. Fabricated TFT operated as n-channel enhancement mode TFT with an on/off ratio of $10^{9}$. The threshold voltage of $4.3 \mathrm{~V}$ and the subthreshold slope of $116 \mathrm{mV} / \mathrm{dec}$ are extracted from $\sqrt{\mathrm{I}_{\mathrm{D}}}-\mathrm{V}_{\mathrm{G}}$ and $\log \left(\mathrm{I}_{\mathrm{D}}\right)-\mathrm{V}_{\mathrm{G}}$ characteristics, respectively.

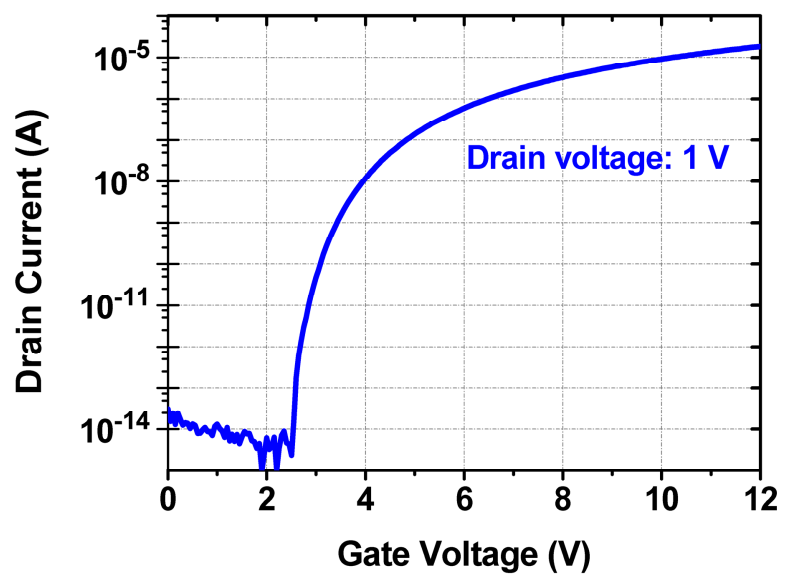

Figure 2. Drain current - gate voltage characteristics of fabricated devices.

\subsection{Trap states of $\mathrm{ZnO}$}

Polycrystalline $\mathrm{ZnO}$ has natural crystallographic defects such as Zinc interstitials and Oxygen vacancies which make $\mathrm{ZnO}$ a naturally n-type doped material [11]. Optical absorption spectrum of 30-nm-thick ZnO, coated on a double side polished quartz substrate, exhibits band to band absorption mechanism and its absorption decreases significantly after the band gap energy (Figure 3). Photoluminescence measurement of this $\mathrm{ZnO}$ film shows broadband emission characteristic around $600 \mathrm{~nm}$ as shown in Figure 3. The distribution of trap states in the band gap is responsible for this broad emission, and the traps in the forbidden band gap are located around $2.07 \mathrm{eV}$ below the conduction band of $\mathrm{ZnO}$. 


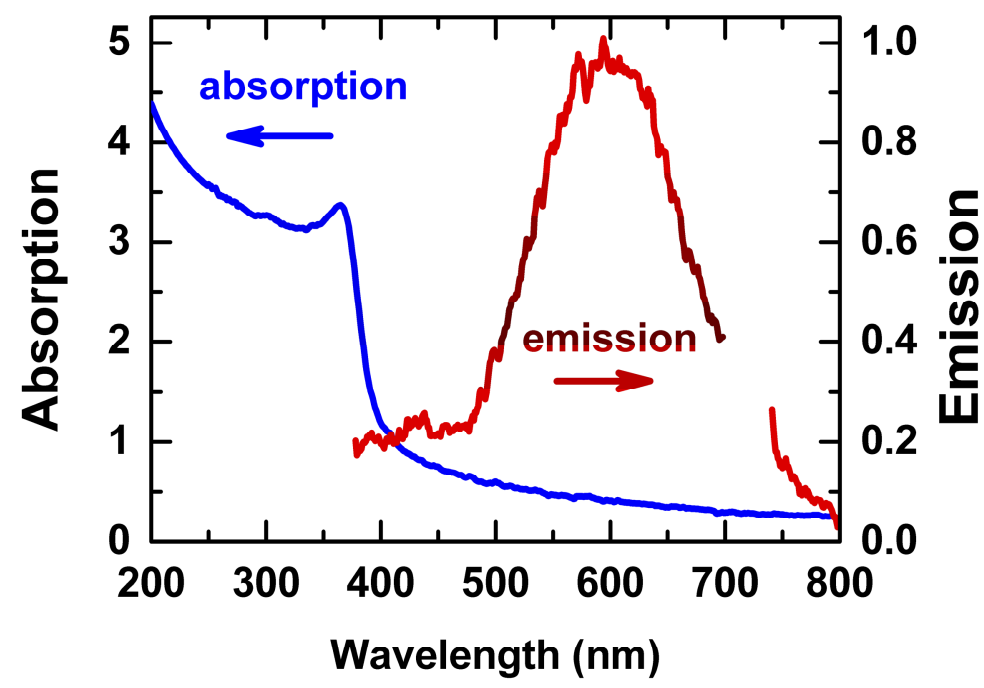

Figure 3. Spectral absorption and emission characteristics of $\mathrm{ZnO}$ deposited on double side polished quartz substrate. $\mathrm{ZnO}$ is optically pumped at $\lambda=350 \mathrm{~nm}$ for the emission measurement.

\section{RESULTS AND DISCUSSION}

\subsection{Tunable absorption of $\mathrm{ZnO}$ channel region}

Optical absorption mechanisms of $\mathrm{ZnO}$ film are shown in Figure 4. Interband mechanism (1) is the absorption of a photon with energy higher than the band gap $(\mathrm{hv}>3.37 \mathrm{eV})$ which results in a transition of an electron from the valence band to the conduction band. For trap-assisted absorption, electron either moves from the valence band to the trap state (2) or from the trap state to the conduction band (3). A photon with energy higher than the energy difference between the trap state and the edge of the valence band $(\mathrm{hv}>1.3 \mathrm{eV})$ excites an electron from the valence band to the trap state energy level, $\mathrm{E}_{\mathrm{T}}$. For this trap-assisted absorption mechanism an unoccupied trap state is required. The third possible absorption mechanism is from the trap state energy level to the conduction band, but spatially localized nature of trap states significantly reduces the probability of such a mechanism.

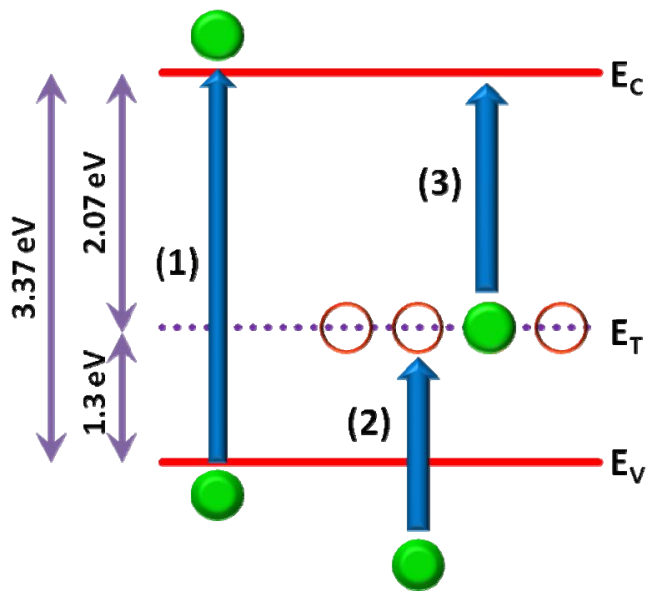

Figure 4. Absorption mechanisms of $\mathrm{ZnO}$. Interband absorption (1). Sub-band gap absorption mechanisms: from valence band to trap state (2), from trap state to conduction band (3).

Responsivity measurements of fabricated ZnO TFTs are taken with the experimental setup shown in Figure 5. The wideband light source is monochromated with Oriel $1 / 8 \mathrm{~m}$ Cornerstone monochromator. The monochrome light is mechanically chopped at $400 \mathrm{~Hz}$ and optically focused on fabricated devices from top-side at normal incidence. Source 
and gate terminals of our TFTs are directly connected to Keithley 2400 sourcemeter whereas drain terminal is connected to the sourcemeter by passing through a lock-in amplifier which measures dynamic photocurrent.

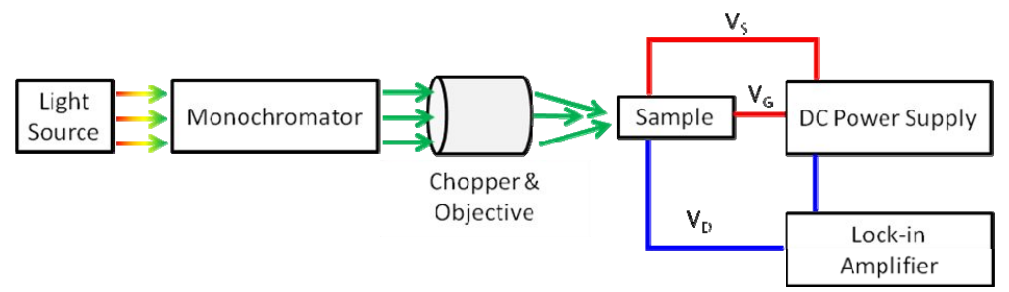

Figure 5. Responsivity measurement setup. Monochromated and mechanically chopped light is focused on fabricated device from top with normal incidence. The photocurrent between drain and source terminals is measured with lock-in amplifier.

In Figure 6, spectral responsivity measurements of our ZnO TFT are given for various gate to source biases and the constant drain to source bias of $3 \mathrm{~V}$. The photoresponse of $\mathrm{ZnO}$ channel layer in the visible spectrum can be actively tuned by controlling gate bias. As it is aforementioned, the sub-band gap absorption mechanism of $\mathrm{ZnO}$ films requires unoccupied trap states. When the ZnO TFT is biased in the depletion region, its traps loose electrons and the number of unoccupied trap states increases. Due to the increasing number of empty trap states, the probability of sub-band gap photon absorption mechanism increases. As $\mathrm{ZnO}$ channel is accumulated with electrons, both its Fermi level and the number of occupied trap states increases.

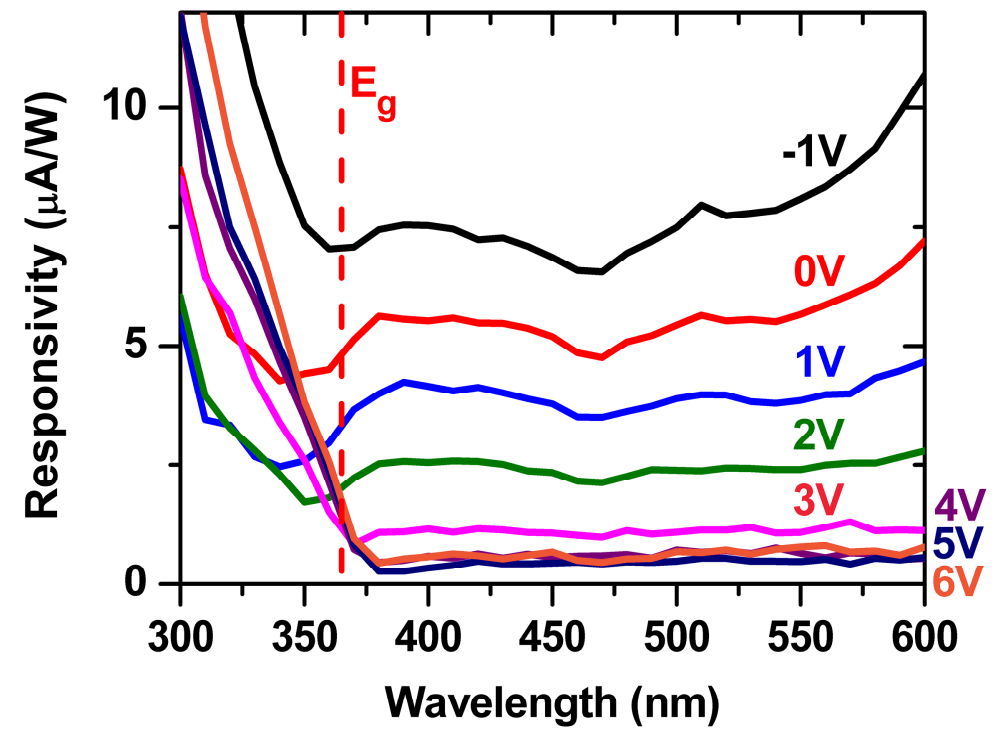

Figure 6. Spectral responsivity measurements of our $\mathrm{ZnO}$ TFT for various gates to source $\left(\mathrm{V}_{\mathrm{GS}}\right)$ biases under the constant drain to source voltage of $3 \mathrm{~V}$. The dashed red line shows cutoff wavelength $(365 \mathrm{~nm})$ of $\mathrm{ZnO}$ band-to-band absorption.

\subsection{Theoretical analysis of absorption coefficient change}

Our ZnO TFT is illuminated from the top-side, and the incident light intensity at the top surface is denoted as $\mathrm{I}_{0}$ (after surface reflection). The light intensity at a distance of ' $x$ ' decreases to $I_{x}$ which is calculated by Eq. 1 where $\alpha$ is the absorption coefficient of $\mathrm{ZnO}$.

$$
\begin{gathered}
\mathrm{I}_{\mathrm{x}}=\mathrm{I}_{0} \cdot \mathrm{e}^{-\alpha \mathrm{x}} \\
\text { Absorption }: \mathrm{I}_{0}-\mathrm{I}_{\mathrm{x}}=\mathrm{I}_{0} \cdot\left(1-\mathrm{e}^{-\alpha \mathrm{x}}\right)
\end{gathered}
$$


The absorption coefficient of $\mathrm{ZnO}$ films at visible wavelengths can be controlled by applying gate bias as it is shown in Figure 6. By assuming the carrier collection efficiency is independent of gate bias, the absorption coefficient ratio is linearly proportional to the responsivity ratio as it is given in Eq. 3. For the wavelength of $550 \mathrm{~nm}$, our ZnO TFT has responsivity values $8.09 \mu \mathrm{A} / \mathrm{W}$ and $0.79 \mu \mathrm{A} / \mathrm{W}$ respectively for depleted $\left(\mathrm{V}_{\mathrm{GS}}=-1 \mathrm{~V}\right)$ and accumulated $\left(\mathrm{V}_{\mathrm{GS}}=6 \mathrm{~V}\right) \mathrm{ZnO}$ TFT, respectively. The absorption ratio given in Eq. 3 can be simplified to ratios of absorption coefficients (Eq. 4) by using Taylor series expansion of exponential term and assuming the product $\left[\begin{array}{l}\text { Absorption } \\ \text { Coefficient }\end{array}\right] \times[$ Depth $](\alpha \mathrm{x})$ is small for both depletion and accumulation cases.

$$
\begin{gathered}
\text { Absorption ratio }: \frac{\mathrm{I}_{0} \cdot\left(1-\mathrm{e}^{-\alpha_{1} \mathrm{x}}\right)}{\mathrm{I}_{0} \cdot\left(1-\mathrm{e}^{-\alpha_{2} \mathrm{x}}\right)}=\frac{8.09 \mu A / W}{0.78 \mu A / W}=10.37 \\
\frac{1-\mathrm{e}^{-\alpha_{1} \mathrm{x}}}{1-\mathrm{e}^{-\alpha_{2} \mathrm{x}}} \approx \frac{1-\left(1-\alpha_{1} \mathrm{x}\right)}{1-\left(1-\alpha_{2} \mathrm{x}\right)}=\frac{\alpha_{1}}{\alpha_{2}}=10.37
\end{gathered}
$$

\subsection{Optical modulator design}

The tunable absorption coefficient mechanism shown in the ZnO TFT device can be applied as an optical modulator in the visible wavelengths by using the proposed modulator design shown in Figure 7. A thin $\mathrm{ZnO}$ film is deposited on a bottom metal contact which is grounded. A thin oxide layer is used to block current flow between contacts and to create an electric field which will deplete or accumulate $\mathrm{ZnO}$ film depending on the applied active modulation bias. The proposed design also improves modulation contrast ratio of thin $\mathrm{ZnO}$ film, by extending the optical path length of incident light in a waveguide configuration.

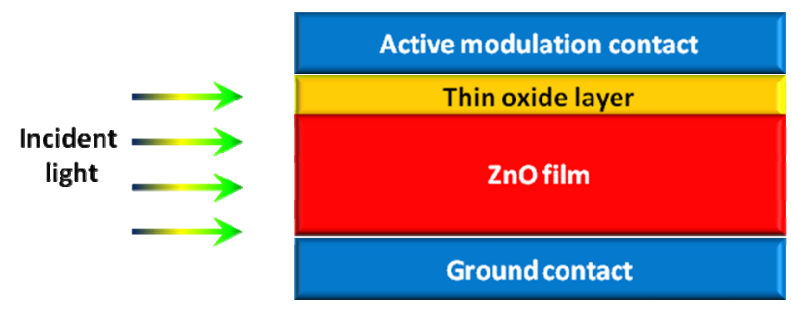

Figure 7. Proposed optical modulator design. The absorption coefficient of $\mathrm{ZnO}$ in the visible spectrum, controlled by applied bias to active modulation contact.

\section{CONCLUSION}

$\mathrm{ZnO}$ channel TFTs are fabricated at $80^{\circ} \mathrm{C}$ by atomic layer deposition technique to demonstrate tunable absorption characteristics of $\mathrm{ZnO}$ layer. The fabricated TFTs exhibited tunable absorption characteristics in the visible light spectrum. As we deplete $\mathrm{ZnO}$ channel an order of magnitude absorption coefficient change is achieved. An optical modulator design which exploits this tunable absorption mechanism is proposed.

\section{ACKNOWLEDGEMENT}

This work was supported in part by European Union Framework Program 7 Marie Curie IRG Grant 239444, COST NanoTP, The Scientific and Technological Research Council of Turkey-TUBITAK Grants 109E044, 112M004 and $112 \mathrm{E} 052$.

\section{REFERENCES}

[1] Wager, J. F., "Transparent Electronics," Science, 300, pp. 1245-1246 (2003).

[2] Fortunato, E., Barquinha, P., and Martins, R., "Oxide Semiconductor Thin-Film Transistors: A Review of Recent Advances," Advanced Materials, 24, pp. 2945-2986 (2012). 
[3] Masuda, S., Kitamura, K., Okumura, Y., Miyatake, S., Tabata, H., and Kawai, T., "Transparent thin film transistors using $\mathrm{ZnO}$ as an active channel layer and their electrical properties," Journal of Applied Physics, 93, pp. 1624-1630 (2003).

[4] Hoffman, R. L., Norris, B. J., and Wager, J. F., "ZnO-based transparent thin-film transistors," Appl. Phys. Lett., 82, pp. 733-735 (2003).

[5] Carcia, P. F., McLean, R. S., Reilly, M. H., and Nunes, G., "Transparent ZnO thin-film transistor fabricated by rf magnetron sputtering," Appl. Phys. Lett., 82, pp. 1117-1119 (2003).

[6] Liang, S., Sheng, H., Liu, Y., Huo, Z., Lu, Y., and Shen, H., "ZnO Schottky ultraviolet photodetectors," Journal of Crystal Growth, 225, pp. 110-113 (2001).

[7] Kind, H., Yan, H., Messer, B., Law, M., and Yang, P., "Nanowire Ultraviolet Photodetectors and Optical Switches," Advanced Materials, 14, pp. 158-160 (2002).

[8] Soci, C., Zhang, A., Xiang, B., Dayeh, S. A., Aplin, D. P. R., Park, J., Bao, X. Y., Lo, Y. H, and Wang, D., "ZnO Nanowire UV Photodetectors with High Internal Gain," Nano Letters, 7, pp. 1003-1009 (2007).

[9] Bae, H. S., Yoon, M. H., Kim, J. H., and Im, S., "Photodetecting properties of ZnO-based thin-film transistors," Appl. Phys. Lett., 83, 5313 (2003).

[10] Wraback, M., Shen, H., Liang, S., Gorla, C. R., and Lu, Y., "High contrast, ultrafast optically addressed ultraviolet light modulator based upon optical anisotropy in $\mathrm{ZnO}$ films grown on R-plane sapphire," Appl. Phys. Lett., 74, 507 (1999).

[11] Guziewicz, E., Godlewski, M., Wachnicki, L., Krajewski, T. A., Luka, G., Gieraltowska, S., Jakiela, R., Stonert, A., Lisowski, W., Krawczyk, M., Sobczak, J. W., and Jablonski, A., "ALD grown zinc oxide with controllable electrical properties," Semiconductor Science and Technology, 27, p. 074011 (2012). 